\title{
MOTIVACIÓN ACADÉMICA Y DESEMPEÑO EN FÍSICA EN UN GRUPO DE ESTUDIANTES DE UN LICEO RUMANO
}

\section{ACADEMIC MOTIVATION AND PERFORMANCE IN PHYSICS IN A GROUP OF STUDENTS FROM A ROMANIAN HIGH SCHOOL}

\author{
Corina Glochner Ruzsa \\ Instituto Teórico “Jean Louis Calderon”, Rumania \\ coriruzs@gmail.com \\ https://orcid.org/0000-0002-5457-0514
}

\begin{abstract}
RESUMEN
El presente estudio, cuantitativo y transversal, tuvo como objetivo determinar si las dimensiones de la motivación académica son predictoras significativos de las calificaciones de fisica de 96 estudiantes de nivel medio superior de una escuela en Rumania, con especialización en matemática e informática. Se utilizaron la Escala de Motivación Académica (AMS HS28) para medir la motivación académica y los registros de calificaciones escolares para medir el desempeño en física. Para el análisis de los datos se utilizó la regresión lineal múltiple. Los resultados mostraron que la motivación intrínseca para el conocimiento es un predictor positivo de las calificaciones de fisica $(\beta=.476, p=.009)$, y que la motivación extrínseca es un predictor negativo $(\beta=-.491, p=.001)$. Se observó también que existe una diferencia significativa entre los dos sexos en la subescala de motivación de inducción extrínseca $(t=2.559, p=.012)$, pues las niñas tuvieron una media de 19.4 y los niños una de 15.8. Se concluye que la motivación intrínseca tiene una relación positiva con el desempeño académico en física, por lo que es necesario que el docente cultive los recursos de la motivación intrínseca permitiendo al mismo tiempo el desarrollo de la autonomía del alumno.
\end{abstract}

Palabras clave: motivación académica, rendimiento académico, desempeño en física

\section{ABSTRACT}

The present study, quantitative and cross-sectional, aimed to determine whether the academic motivation dimensions are significant predictors of the physics grades of 96 high school students from a school in Romania specializing in mathematics and computing. The Academic Motivation Scale (AMS HS-28) was used to measure academic motivation, and school grade records were used to measure physics performance. For data analysis, multiple linear regression was used. The results showed that intrinsic motivation for knowledge is a positive predictor of physics grades $(\beta=.476, p=.009)$, 


\section{MOTIVACIÓN ACADÉMICA Y RENDIMIENTO}

and that extrinsic motivation is a negative predictor $(\beta=-.491, p=.001)$. It was also observed that there is a significant difference between the two sexes in the extrinsic induction motivation subscale $(t=2.559, p=.012)$, since the girls had a mean of 19.4 and the boys one of 15.8. It is concluded that intrinsic motivation has a positive relationship with academic performance in physics, so it is necessary for the teacher to cultivate intrinsic motivation resources while allowing the development of student autonomy.

Keywords: academic motivation, academic performance, performance in physics

\section{Introducción}

La alfabetización científica es cada vez más importante para la sociedad tecnológica actual (Organisation for Economic Co-operation and Development [OECD], 2007). Sin embargo, en las últimas dos décadas se ha visto una disminución en el número de estudiantes interesados en la ciencia. Para muchos maestros es un verdadero desafío mantener el entusiasmo de los estudiantes por la escuela y la motivación para aprender (Denzine y Brown, 2015). En estas condiciones, es muy importante que los docentes utilicen las mejores estrategias para motivar a los estudiantes (Palmer, 2007).

La educación superior tiene como objetivo animar a los estudiantes a alcanzar un mayor nivel de comprensión de las materias estudiadas. Para que los estudiantes alcancen un nivel superior, deben aprender en profundidad (Barab y Plucker, 2002) y estar motivados para lograr este desempeño (Deci y Ryan, 1985).

La motivación juega un papel importante en casi todos los procesos humanos de toma de decisiones. Independientemente de la situación, poco se puede lograr sin una capitalización adecuada de la motivación (Schatt, 2011). Los profesores tienen un papel importante en la motivación de los estudiantes.
Sin duda, es mucho más fácil decirle a los alumnos que el agua hierve a 212 grados Fahrenheit, pero implica un mayor esfuerzo realizar un experimento en el que observen qué sucede cuando la temperatura del agua en la olla sobre la estufa alcanza esta temperatura (Knotts et al., 2004). Es necesario despertar un espíritu de investigación y los profesores deben ayudar a los estudiantes a pensar y lograr ciertas cosas prácticas útiles (White, 2000).

Blomster (citado en Bacon, 2012) afirma que la motivación no siempre es algo que se puede observar o medir directamente, sino que se puede estimular. El papel de los profesores es animar y estimular a los estudiantes para que tengan éxito y la clave es la motivación. Dada la importancia de la ciencia para el futuro de los estudiantes, los sistemas educativos deben garantizar que los estudiantes tengan tanto el interés como la motivación para continuar aprendiendo, incluso después de terminar la escuela (OECD, 2007).

Estudios previos han mostrado que existen varios factores que influyen en las calificaciones de los estudiantes, tales como pocas horas de sueño durante la noche (BaHammam et al., 2012), asistencia a clases (Thatcher et al., 2007), esfuerzo del estudiante (Goodman et al., 2011), estrategias de enseñanza (Soto 
y Anand, 2009), el padre helicóptero (Schiffrin y Liss, 2017) y la motivación (Morales Rodríguez, 2011).

\section{La motivación}

La motivación es uno de los conceptos psicológicos más importantes (Guay et al., 2008). Tiene un impacto positivo en el aprendizaje (Lopes et al., 2018) y en el rendimiento académico (Wagner y Szamosközi, 2012), a la vez que estimula, apoya y dirige una actividad (Niemiec y Ryan, 2009).

El concepto de motivación se puede definir como la fuerza interna que estimula el entusiasmo, la perseverancia y determina al individuo a actuar, pensar y continuar una conducta desde el momento en que fue elegida. Es la fuerza que moviliza al individuo a actuar con el fin de alcanzar una meta (Deci y Ryan, 2008a; Ghazi et al., 2010; Pi-Yueh et al., 2011; Singh et al., 2011).

Los estudiantes que están fuertemente interesados están motivados, aprenden y tienen éxito (Linnenbrink y Pintrich, 2002; Aladağ, 2010).

La motivación energiza el comportamiento de un individuo, guía el comportamiento hacia un objetivo específico, ayuda a acumular conocimientos, desarrolla la capacidad de comunicarse en la sociedad, aumenta el deseo de perseverar en las actividades, conduce al desempeño y desarrolla un sentido de disciplina en el individuo (Singh et al., 2011).

En el pasado, la teoría de la motivación se ha abordado principalmente como un concepto unidimensional (Deci y Ryan, 2008b). La teoría de la autodeterminación comenzó diferenciando la motivación y sosteniendo que la motivación académica tiene un carácter multidimensional (Areepattamannil, 2014). En el contexto educativo, se han propuesto tres tipos de motivación conducente a la autodeterminación continua (Stover et al., 2014): motivación intrínseca, motivación extrínseca y desmotivación (Deci y Ryan, 2000; Deci y Ryan, 2008b).

La motivación intrínseca, en general, se refiere al deseo de participar en una actividad porque esta actividad es placentera y despierta una sensación de satisfacción (Ali et al., 2011; Deci y Ryan, 2000; Noels et al., 2003). Un estudiante está intrínsecamente motivado cuando va a clase y considera que lo que está aprendiendo es interesante y satisfactorio (Vallerand et al., 1992). Este tipo de motivación se considera una base importante para el aprendizaje (Niemiec y Ryan, 2009).

No todos los comportamientos están motivados intrínsecamente; algunos de ellos son estimulados por factores externos (Dincer y Yesilyurt, 2017). A diferencia de los comportamientos intrínsecamente motivados, los extrínsecamente motivados se inician por estímulos externos, tales como competencias grupales (Noels et al., 2003), tareas para obtener una recompensa prometida (Pi -Yueh et al., 2011) o evitación del castigo (Noels et al., 2003).

Los estudiantes pueden estar motivados extrínsecamente para aprender ciencia cuando se dan cuenta de que este conocimiento será útil para estudios futuros o profesionales (OECD, 2007). Sin embargo, la motivación extrínseca solo conduce a resultados a corto plazo, pero no es eficaz a largo plazo (Levpuscek et al., 2012). A diferencia de los estudiantes motivados extrínsecamente, los estudiantes motivados intrínsecamente tienden a aprender más profundamente y son más creativos porque usan su tiempo y energía por su propia iniciativa para estudiar (Niemiec y Ryan, 2009). 
Estos dos tipos de comportamiento, los que responden a factores externos como los que lo hacen a factores internos al individuo, afectan la motivación de la persona (Vallerand, 2012).

Un concepto final de la teoría de la autodeterminación es la desmotivación (Guay et al., 2003). La desmotivación se refiere a la situación en la que las personas no ven ninguna conexión entre sus acciones y sus consecuencias. Las consecuencias se consideran el resultado de factores sobre los que no tienen control (Abramson et al., como se cita en Noels et al., 2003). Las personas desmotivadas carecen de energía motivacional, no tienen un propósito y se perciben a sí mismas como incapaces de revertir el curso de los acontecimientos (Guay et al., 2003; Stover et al., 2014).

La motivación académica es un factor muy importante en el proceso de aprendizaje que conduce a buenos resultados escolares. Este estudio buscó conocer si las dimensiones de la motivación académica son predictores significativos de las calificaciones en física de los estudiantes de un liceo en Rumania, con especialización en matemática e informática.

\section{Método \\ Tipo de investigación}

La presente investigación tuvo un enfoque cuantitativo, ex post facto. Debido a que solo se realizó una medición de las variables en un tiempo determinado, el estudio fue de tipo transversal.

\section{Participantes}

Participaron del estudio 96 estudiantes de los grados IX-XII de un liceo especializado en matemática e informática en Rumania, 36 de sexo femenino y 60 de sexo masculino.

\section{Instrumentos}

Motivación académica. Se utilizó la Escala de Motivación Académica versión secundaria (AMS-HS 28) para determinar el tipo de motivación. El instrumento fue traducido del inglés al rumano. La herramienta consta de 28 ítems calificados en una escala Likert de siete puntos. Contiene siete subescalas: tres de motivación intrínseca (conocimiento, logro y estimulación), tres de motivación extrínseca (identificación, inducción y regulación externa) y una de desmotivación, cada una con cuatro preguntas. Los coeficientes de confiabilidad alfa de Cronbach obtuvieron valores de entre .83 y .86 , excepto en la motivación extrínseca, donde obtuvo un valor de .62 (Vallerand et al., 1992). Para el instrumento traducido al rumano el coeficiente alfa de Cronbach tuvo valores entre .76 y .89 .

Rendimiento académico. Los datos del desempeño académico en física se obtuvieron de los registros de calificaciones correspondientes al año escolar 2017-2018. El rango de calificaciones va del 5 al 10, donde 5 es la puntuación más baja y 10 la más alta.

\section{Análisis de datos}

Para saber si había una correlación significativa entre las dimensiones de la motivación académica y las calificaciones de física de los estudiantes, se utilizó el método de regresión lineal múltiple.

\section{Resultados \\ Análisis descriptivo \\ La principal dimensión de la moti- vación académica para la obtención de buenos resultados en física de los estu- diantes fue la motivación extrínseca de regulación externa $(M=23.2)$, lo que significa que la principal motivación}


proviene de obtener resultados tangibles, como obtener un título. Como se muestra en la Tabla 1, le sigue la motivación intrínseca para el conocimiento $(M=19.6)$ y la motivación externa por identificación $(M=19.3)$. La desmo- tivación se coloca en último lugar ( $M$ $=8.4$ ).

La puntuación más baja obtenida para el promedio anual en física fue de 6 y la más alta de 10 , donde el mínimo posible es 5 y el máximo 10 .

Tabla 1

Estadistica descriptiva de las dimensiones de la motivación académica $(\mathbb{N}=96)$

\begin{tabular}{lccccc}
\hline & \multicolumn{3}{c}{$\begin{array}{c}\text { Rango de valores } \\
\text { observado }\end{array}$} & & \\
\cline { 2 - 3 } \multicolumn{1}{c}{ Subescala } & Mínimo & Máximo & & $M$ & DE \\
\hline Motivación intrínseca - conocimiento & 4 & 28 & 19.6 & 5.168 \\
Motivación intrínseca - logro & 4 & 28 & 17.4 & 5.803 \\
Motivación intrínseca - estimulación & 4 & 28 & 16.0 & 5.329 \\
Motivación - identificación extrínseca & 4 & 28 & 19.3 & 5.945 \\
Motivación - inducción extrínseca & 4 & 28 & 17.1 & 6.952 \\
Motivación extrínseca - regulación externa & 4 & 28 & 23.2 & 4.674 \\
Desmotivación & 4 & 28 & 8.4 & 1.662 \\
\hline
\end{tabular}

\section{Análisis correlacional}

El análisis de regresión lineal múltiple mostró que dos de las dimensiones de la motivación académica aportan significativamente a la varianza del desempeño en física $\left(R=.318, R^{2}\right.$ corregida $=$ $\left..082, F_{(2.39)}=5.239, p=.007\right)$.

El modelo predictor está conformado por las dos dimensiones que se señalan a continuación: la motivación intrínseca por conocimiento, que tiene una fuerte influencia positiva en las calificaciones de física con un coeficiente $(\beta=.286$, $p=.014)$, y la motivación por identificación extrínseca, que tiene una fuerte influencia negativa en las calificaciones de física con un coeficiente $(\beta=-.346$, $p=.003)$.

\section{Diferencia por sexo}

En la dimensión de motivación académica por inducción extrínseca, las ni- ñas registraron una media significativamente más alta $(M=19.4)$ que los niños $(M=15.8)$. Los resultados obtenidos $(t$ $(76,566)=2,559, p=.012)$ mostraron que existe una diferencia significativa entre los dos sexos en esta subescala.

\section{Discusión}

Con base en el análisis estadístico, se observó que existe una diferencia significativa entre los dos sexos en la subescala de motivación por inducción extrínseca. Así, se encontró que este tipo de motivación se presenta más en las niñas que en los niños, lo que significa que las niñas quieren mostrar que son inteligentes, capaces de aprobar exámenes importantes como el bachillerato, quieren sentirse importantes y quieren demostrar que pueden tener éxito en la escuela. Esto puede explicarse por el hecho de que, durante el período comunista que atravesó 
Rumania, las mujeres estaban representadas, como dice Einhorn (1995), como una entidad anónima, una representación alegórica que más bien simboliza cualidades abstractas e ideal que un ser vivo.

Las representaciones de la mujer en este período presentan una realidad ideal, como se pretendía que fuera, no como realmente era. Las mujeres estaban marginadas, estaban demasiado ocupadas con las tareas del hogar, con el trabajo, con la crianza de sus hijos y sin acceso a la educación. Esto ha llevado al deseo de estas mujeres de que al menos sus hijas sean vistas de manera diferente en la sociedad y tengan acceso a la educación. Así, a las niñas de esta generación se les inculcó el deseo de salir del anonimato, lo que aumentó su nivel de motivación por inducción extrínseca.

Por otro lado, existe la mentalidad de que los niños son mejores en materias exactas y las niñas son mejores en las humanas. Esta hipótesis ha significado que en los últimos cinco años incluso ha habido una Olimpiada Matemática Europea separada para niñas y niños. En este contexto, se entiende por qué las niñas de la especialidad matemática-informática quieren tener éxito, quieren demostrar que son inteligentes y que pueden tener éxito en la escuela.

El resultado de esta investigación es similar al obtenido por Cavas (2011). Su estudio mostró que la motivación para aprender ciencias difiere según el gé- nero. Las niñas estaban más motivadas para aprender ciencia que los niños. Sin embargo, este estudio no especifica qué tipo de motivación es más importante para las niñas.

El análisis de regresión múltiple a partir de la correlación de las notas de estudio/promedio anual en física y los siete tipos de motivación académica reveló que dos de ellos constituyen el modelo predictor. Se observó un aporte significativo de la motivación intrínseca por conocimiento sobre las calificaciones en física, mientras que la motivación extrínseca por identificación mostró una influencia negativa sobre ellas.

Estos resultados son consistentes con otros estudios que muestran que la motivación intrínseca y la motivación extrínseca fueron predictores significativos de los logros matemáticos de los adolescentes inmigrantes indios en $\mathrm{Ca}$ nadá, donde la motivación intrínseca fue un predictor positivo significativo de las calificaciones de matemáticas, mientras que la motivación extrínseca fue un predictor negativo. La desmotivación no fue un predictor significativo de las calificaciones en matemáticas para los adolescentes indios inmigrantes en Canadá (Areepattamannil, 2014).

Los hallazgos de este estudio contribuyen a la evidencia ya existente sobre la importancia de que los docentes promuevan la motivación intrínseca de los estudiantes por sus efectos favorables sobre su desempeño académico.

\section{Referencias}

Aladağ, E. (2010). The effects of GIS on students' academic achievement and motivation in seventhgrade social studies lessons in Turkey. International Research in Geographical and Environmental Education, 19(1), 11-23. https://doi.org/10.1080/10382040903545476

Areepattamannil, S. (2014). Relationship between academic motivation and Mathematics achievement among Indian adolescents in Canada and India. Journal of General Psychology, 141(3), 247-262. https://doi.org/10.1080/00221309.2014.897929

Bacon, A. (2012). Mathematical student motivation. https://eric.ed.gov/?id=ED528937 


\section{GLOCHNER RUZSA}

BaHammam, A. S., Alaseem, A. M., Alzakri, A. A., Almeneessier, A. y Sharif, M. M. (2012). The relationship between sleep and wake habits and academic performance in medical students: A crosssectional study. BMC Medical Education, 12(61). https://doi.org/10.1186/1472-6920-12-61

Barab, S. A. y Plucker, J. A. (2002). Smart people or smart contexts? Cognition, ability, and talent development in an age of situated approaches to knowing and learning. Educational Psychologist, 37(3), 165-182. https://doi.org/10.1207/S15326985EP3703 3

Cavas, P. (2011). Factors affecting the motivation of Turkish primary students for science learning. Science Education International, 22(1), 31-42. http://www.icaseonline.net/sei/march2011/p3.pdf

Deci, E. L. y Ryan, R. M. (1985). Intrinsic motivation and self-determination in human behavior. Plenum.

Deci, E. L. y Ryan, R. M. (2000). The "what" and "why" of goal pursuits: Human needs and the selfdetermination of behavior. Psychological Inquiry, 11(4), 227-268. https://doi.org/10.1207/ S15327965PLI1104_01

Deci, E. L. y Ryan, R. M. (2008a). Facilitating optimal motivation and psychological well-being across life's domains. Canadian Psychology, 49(1), 14-23. https://doi.org/10.1037/07085591.49.1.14

Deci, E. L. y Ryan, R. M. (2008b). Self-determination theory: A macrotheory of human motivation, development, and health. Canadian Psychology, 49(3), 182-185. https://doi.org/10.1037/a0012801

Denzine, G. y Brown, R. (2015). Motivation to learn and achievement. En R. Papa (Ed.), Media rich instruction: Connecting curriculum to all learners (pp. 19-34). Springer. https://doi.org/10.1007/978-3-319-00152-4_2

Dincer, A. y Yesilyurt, S. (2017). Motivation to speak English: A self-determination theory perspective. PASAA: Journal of Language Teaching and Learning in Thailand, 53, 1-25. http://www.culi.chula. ac.th/publicationsonline/files/article/qSSPiyUzqfMon110323.pdf

Einhorn, B. (1995). Cinderalla goes to market: Citizenship, gender, and women's movements in East Central Europe (Book). American Journal of Sociology, 100(5), 1343-1345. https://doi.org/10.1086/230649

Ghazi, S. R., Ali, R., Shahzad, S. y Hukamdad, H. (2010). Parental involvement in children academic motivation. Asian Social Science, 6(4), 93-99. https://doi.org/10.5539/ass.v6n4p93

Goodman, S., Jaffer, T., Keresztesi, M., Mamdani, F., Mokgatle, D., Musariri, M., Pires, J. y Schlechter, A. (2011). An investigation of the relationship between students' motivation and academic performance as mediated by effort. South African Journal of Psychology, 41(3), 373-385. https://doi. org $/ 10.1177 / 008124631104100311$

Guay, F., Mageau, G. A. y Vallerand, R. J. (2003). On the hierarchical structure of selfdetermined motivation: A test of top-down, bottom-up, reciprocal, and horizontal effects. Personality and Social Psychology Bulletin, 29(8), 992-1004. https://doi.org/10.1177/0146167203253297

Guay, F., Marsh, H. W., Senécal, C. y Dowson, M. (2008). Representations of relatedness with parents and friends and autonomous academic motivation during the late adolescence-early adulthood period: Reciprocal or unidirectional effects? British Journal of Educational Psychology, 78(4), 621-637. https://doi.org/10.1348/000709908X280971

Knotts, H., Livingston, C., Mercer, G. E. y Friedman, B. D. (2004). Integrating teaching, learning, and community outreach: Western Carolina's local government youth assembly. International Social Science Review, 79(1/2), 56-62. https://www.jstor.org/stable/41887169

Levpuscek, M. P., Zupancic, M. y Socan, G. (2012). Predicting achievement in Mathematics in adolescent students: The role of individual and social factors. The Journal of Early Adolescence, 33(4), 523-551. https://doi.org/10.1177/0272431612450949

Linnenbrink, E. A. y Pintrich, P. R. (2002). Motivation as an enabler for academic success. School Psychology Review, 31(3), 313-327. https://doi.org/10.1080/02796015.2002.12086158

Lopes, P., Silva, R., Oliveira, J., Ambrósio, I., Ferreira, D., Crespo, C. y Rosa, P. J. (2018). Rasch analysis on the academic motivation scale in Portuguese University Students. NeuroQuantology, 16(3), 41-46. https://doi.org/10.14704/nq.2018.16.3.1062

Morales Rodríguez, F. M. (2011). Aprendizaje, motivación y rendimiento en estudiantes de lengua extranjera inglesa. Psicología Educativa, 17(2), 195-207. https://doi.org/10.5093/ed2011v17n2a6

Niemiec, C. P. y Ryan, R. M. (2009). Autonomy, competence, and relatedness in the classroom: Applying self-determination theory to educational practice. Theory and Research in Education, 7(2), 133-144. https://doi.org/10.1177/1477878509104318

Noels, K. A., Pelletier, L. G., Clément, R. y Vallerand, R. J. (2003). Why are you learning a second language? Motivational orientations and self-determination theory. Language Learning, 53(S1), 33-64. https:// doi.org/10.1111/1467-9922.53223

Organisation for Economic Co-operation and Development. (2007). PISA 2006 science competencies for tomorrow's world. https://www.oecd.org/unitedstates/39722597.pdf 


\section{MOTIVACIÓN ACADÉMICA Y RENDIMIENTO}

Palmer, D. (2007). What is the best way to motivate students in science? Teaching Science, 53(1), 38-42. https://novaprd-lb.newcastle.edu.au/vital/access/manager/Repository/uon:9948

Pi-Yueh, C., Mei-Lan, L. y Chia-Kai, S. (2011). Attitudes and motivations of students taking professional certificate examinations. Social Behavior and Personality, 39(10), 1303-1314. https://oi.org/10.2224/ sbp.2011.39.10.1303

Schatt, M. D. (2011). Achievement motivation and the adolescent musician: A synthesis of the literature. Research and Issues in Music Education, 9(1), Artículo 5. https://commons.lib.jmu.edu/rime/vol9/iss1/5/

Schiffrin, H. y Liss, M. (2017). The effects of helicopter parenting on academic motivation. Journal of Child and Family Studies, 26(5), 1472-1480. https://doi.org/10.1007/s10826-017-0658z

Singh, S., Singh, A. y Singh, K. (2011). Academic motivation among urban and rural students: A study on traditional vs open education system in India. Turkish Online Journal of Distance Education, 12(4), 133-146. https://dergipark.org.tr/en/pub/tojde/issue/16906/176300

Soto, J. G. y Anand, S. (2009). Factors influencing academic performance of students enrolled in a lower division cell biology core course. Journal of the Scholarship of Teaching and Learning, 9(1), 64-80. https://scholarworks.iu.edu/journals/index.php/josotl/article/view/1720

Stover, J. B., Uriel, F., de la Iglesia, G., Freiberg Hoffmann, A. y Fernández Liporace, M. (2014). Rendimiento académico, estrategias de aprendizaje y motivación en alumnos de Escuela Media de Buenos Aires. Perspectivas en Psicología, 11(2), 10-20. http://200.0.183.216/revista/index.php/pep/ article/view/159

Thatcher, A., Fridjhon, P. y Cockcroft, K. (2007). The relationship between lecture attendance and academic performance in an undergraduate psychology class. South African Journal of Psychology, 37(3), 656660. https:/doi.org/10.1177/008124630703700316

Vallerand, R. J. (2012). From motivation to passion: In search of the motivational processes involved in a meaningful life. Canadian Psychology, 53(1), 42-52. https://doi.org/10.1037/a0026377

Vallerand, R. J., Pelletier, L. G., Blais, M. R., Briere, N. M., Senecal, C. y Vallieres, E. F. (1992). The Academic Motivation Scale: A measure of intrinsic, extrinsic, and amotivation in education. Educational and Psychological Measurement, 52(4), 1003-1017. https://doi.org/10.1177/001316449 2052004025

Wagner, E. y Szamosközi, Ş. (2012). Effects of direct academic motivation-enhancing intervention programs: A meta-analysis. Journal of Cognitive and Behavioral Psychotherapies, 12(1), 85-101. http://jebp.psychotherapy.ro/vol-xii-no-1-2012/effects-direct-academic-motivation-enhancingintervention-programs-meta-analysis/

White, E. G. (2000). Minte, caracter, personalitate. Viață și Sănătate.

Recibido: 13 de julio de 2020

Revisado: 17 de noviembre de 2020

Aceptado: $1^{\circ}$ de diciembre de 2020 